\section{BMJ Paediatrics Open}

\title{
Survey of the practices of neonatologists in managing neonates believed to be at high risk of sleep disordered breathing
}

\author{
Bhavesh Mehta (D , ${ }^{1}$ Karen Waters, ${ }^{2}$ Dominic Fitzgerald, ${ }^{3}$ Nadia Badawi ${ }^{1}$
}

To cite: Mehta B, Waters $\mathrm{K}$, Fitzgerald $\mathrm{D}$, et al. Survey of the practices of neonatologists in managing neonates believed to be at high risk of sleep disordered breathing. BMJ Paediatrics Open 2021;5:e000979. doi:10.1136/ bmjpo-2020-000979

Received 5 December 2020 Revised 28 January 2021 Accepted 30 January 2021
Check for updates

(c) Author(s) (or their employer(s)) 2021. Re-use permitted under CC BY-NC. No commercial re-use. See rights and permissions. Published by BMJ.

${ }^{1}$ Neonatology, Children's Hospital at Westmead, Westmead, New South Wales, Australia

${ }^{2}$ Sleep Medicine, Children's Hospital at Westmead, Westmead, New South Wales, Australia

${ }^{3}$ Respiratory Medicine, Children's Hospital at Westmead, Westmead, New South Wales, Australia

Correspondence to Dr Bhavesh Mehta; bhavesh. mehta@health.nsw.gov.au

\section{ABSTRACT}

Objective To determine the practices of neonatologists in managing high-risk neonates believed to be at risk of sleep disordered breathing (SDB).

Design An electronic web-based questionnaire assessing awareness of and current practices for managing neonates predisposed to SDB with conditions like craniofacial anomalies, neuromuscular disorders or airway problems was emailed to 232 neonatologists and neonatal fellows working in Australia and New Zealand (NZ). Additionally, neonatologists were approached directly during the annual Australia and NZ perinatal conference in 2019.

Results 93 neonatologists (40\%) responded to the survey. The majority (85\%) of the respondents stated that there were no written protocols/criteria for sleep consultation in their unit. We found considerable variations in the threshold for performing tests including oximetry or referring for polysomnography. Most respondents would perform oximetry before referring for a sleep consultation. However, the duration of oximetry varied from 6 to 24 hours and there was no consensus about what is considered abnormal on an oximetry study.

Conclusion Management of SDB is gaining importance in neonatal care because of prolonged length of hospital stay and possible long-term effects of SDB. Responses received suggest a lack of clarity regarding thresholds for referral for treatment of SDB. Likely contributory factors are concerns regarding a lack of long-term outcome data from treatment perceived to be onerous for the family, unsettling to some infants and delaying hospital discharge. To overcome inconsistencies in practice, standardised guidelines for assessing and managing SDB in neonates are needed.

\section{BACKGROUND}

Sleep disordered breathing (SDB) is increasingly recognised as a cause of morbidity and prolonged hospital stay in neonates and young infants. SDB involves a spectrum of problems in neonates ${ }^{1}$; the most severe form is obstructive sleep apnoea (OSA), which causes obstructive events and associated arousals leading to impaired sleep quality and disturbances in both oxygen saturation and carbon dioxide $\left(\mathrm{CO}_{2}\right)$.

Neonates with OSA often suffer recurrent oxygen desaturation and $\mathrm{CO}_{9}$ retention (type II respiratory failure). Previous

\section{What is known about the subject?}

- Sleep disordered breathing (SDB) can have longterm effects in children if not treated.

- There is a lack of clear diagnostic criteria and therapeutic threshold in neonates and young infants.

\section{What this study adds?}

Management practices for neonates with suspected SDB vary considerably.

- There is a lack of guideline for management, and decisions are mostly physician dependent.

- Higher level of evidence is needed to standardise the practices and care of this group of neonates.

studies have demonstrated an association between episodes of hypoxia and poor growth and long-term neurodevelopmental delay. ${ }^{2}$ SDB has been linked to several longterm adverse effects in children including hypertension, ${ }^{3}$ behavioural problems ${ }^{4}$ and impairment of cognition, attention and executive functions. ${ }^{56}$ Therefore, early identification of high-risk infants is important so that treatment can improve quality of life and neurocognitive function. ${ }^{78}$ There is a gap in literature regarding SDB in neonates and its long-term outcomes. Piteo et a t $^{\dagger}$ reported that infants who snored had lower cognitive scores compared with controls. In a study of 33 infants with cleft lip/palate, Smith et $a l^{10}$ reported that infants with more obstructive episodes had lower global behaviour scores at 3 years of age. However, a couple of recent studies showed negative links between SDB in neonates and long-term neurodevelopmental outcomes. ${ }^{11} 12$

Neonates are more vulnerable to OSA due to number of reasons including anatomical considerations (small mid-face), obligate nasal breathing, immature respiratory control and predominance of rapid eye movement sleep state, which may exacerbate obstruction due to loss of muscle tone. In young children, 
adenotonsillar hypertrophy is the principal cause of OSA. However, in infants, anatomical anomalies are the predominant cause like laryngomalacia, macroglossia, craniofacial disorders such as craniosynostosis (Crouzon's disease, Apert syndrome and Pfeiffer syndrome), cleft palate and Pierre-Robin sequence, infants with mid-face hypoplasia (eg, Treacher Collins and Goldenhar syndromes) and with choanal atresia (CHARGE association) $\cdot{ }^{13-17}$ Low muscle tone is also an important predisposing factor, for example, in infants with Down syndrome, Prader-Willi syndrome or neuromuscular disorders. ${ }^{14}$

Polysomnography (PSG) remains the gold standard test to diagnose and quantify the severity of SDB. It uses a combination of measurements including electroencephalogram, electro-oculogram for eye movements and submental electromyogram for muscle tone to characterise the sleep architecture. Respiratory function is assessed by nasal air flow, chest and abdomen movements, oximetry and transcutaneous $\mathrm{CO}_{2}$ level. In addition to that, heart rate, limb movements, audio sounds to detect snoring and video recordings are also monitored.

However, PSGs in infants can only be performed at specialised paediatric centre in consultation with a paediatric sleep physician. There is usually a prolonged wait time for admission in the accepting centre due to a combination of bed constraints and availability of sleep technicians to perform polysomnograms in the neonatal intensive care unit (NICU) which leads to longer hospital stay.

Overnight oximetry study has been used as a screening tool in children and adults; however, there are limited data in young infants. An oximetry study includes monitoring of heart rate, respiratory rate and oxygen saturation. However, its sensitivity depends on the severity of SDB and the presence of more significant oxygen desaturation. Normal oximetry does not rule out SDB and therefore does not replace PSG. A high prevalence of movement artefacts also makes data interpretation challenging. A recent study showed good correlation between the desaturation index obtained from an overnight oximetry study to the Apnoea-Hypopnoea Index (AHI) obtained from PSG in young infants $(<6$ months) at risk of SDB. ${ }^{18}$

The exact prevalence of SDB in neonates is unknown. However, its recognition seems to be increasing. ${ }^{12} 1920$ Currently, in the absence of clinical guidelines, our experience in the largest surgical neonatal unit in New South Wales is that the approach to assessing a newborn with suspected SDB in both regional and tertiary settings varies significantly and in effect is largely driven by the experience of individual clinician.

Consequently, the aim of our study was to assess the knowledge and awareness of SDB in neonatologists and neonatal fellows working across Australia and New Zealand (NZ), and to determine their practices and referral criteria for sleep consultation.

\section{METHODS}

A questionnaire (online supplemental appendix 1) was devised in consultation with paediatric sleep physicians, with questions tailored to suit the clinical picture of neonates seen in our practice. A pilot survey was first sent for feedback to four neonatologists, experienced in managing neonates with SDB who were excluded from the final survey. The final questionnaire had 17 multiple choice questions and respondents could pick one or more answers as necessary (free text was available for additional comments).

In the survey, we asked a series of questions to explore knowledge and practices of neonatologists regarding the management of neonates predisposed to SDB in various conditions including craniofacial anomalies, syndromes like Down and Beckwith-Wiedemann syndromes, neuromuscular disorders, functional anomalies of airway and in preterm infants with moderate-severe chronic lung disease. Symptoms were arranged in a Likert-type scale and respondents were asked to choose a level/symptom at which they would recommend oximetry or initiate referral for PSG. By asking about timing for referral for PSG, we were trying to ascertain timing for sleep consultations as the PSG needs to be conducted and interpreted in specialised centres by the sleep physicians.

\section{Patient and public involvement}

Patients or members of the public were not involved in the design or conduct of the study.

\section{Participants}

This study was conducted in two phases. In the first phase, a web-based questionnaire was emailed to 232 neonatologists and neonatal fellows working across Australia and NZ (contact information was taken from Australia and NZ Neonatal Network Directory 2017). The survey was created using the online software Survey Monkey. The email contained a paragraph explaining the study with a link to the survey. The survey was open for 8 weeks and two reminder emails were sent in this period. In the second phase, we approached neonatologists at the annual perinatal conference of Australia and NZ in 2019 to complete the survey. The identity of physicians answering the questionnaire was kept confidential throughout the data collection, analysis and presentation of results.

No financial incentive was offered for taking part in the survey. Descriptive analysis was performed using Microsoft Excel, and data are presented as a number or percentage.

\section{RESULTS}

Seventy-three responses were received from the online survey, with a further 20 more responses in phase 2, making a total of 93 responses $(40 \%$ response rate). The majority of responses $(80 \%)$ were from neonatologists working in a major NICU (centres providing care for 
Table 1 Neonatologists' practices about SDB in neonates

\begin{tabular}{|c|c|c|}
\hline Responses & $\mathbf{N}$ & $\%$ \\
\hline \multicolumn{3}{|c|}{$\begin{array}{l}\text { 1. How many neonates have you referred for sleep } \\
\text { consultation in the last } 12 \text { months? }\end{array}$} \\
\hline a. 0 & 18 & 19 \\
\hline b. $1-3$ & 38 & 41 \\
\hline c. $4-6$ & 6 & 7 \\
\hline d. $>6$ & 31 & 33 \\
\hline
\end{tabular}

2. Clinical features suggestive of significant SDB in a term newborn (multiple options could be selected):

\begin{tabular}{|c|c|c|}
\hline $\begin{array}{l}\text { a. Bradycardia in the absence of apnoea or } \\
\text { desaturation }\end{array}$ & 17 & 18 \\
\hline b. Apnoea and desaturations & 64 & 69 \\
\hline $\begin{array}{l}\text { c. Multiple desaturations on overnight } \\
\text { oximetry }\end{array}$ & 62 & 67 \\
\hline d. Desaturation during feeding & 16 & 17 \\
\hline e. All of the above & 26 & 28 \\
\hline
\end{tabular}

SDB, sleep disordered breathing.

high-risk newborns with complex and critical conditions and providing state-wide specialist services) and majority of them were working in a perinatal unit $(67 \%)$.

A decision to refer for sleep consultation was taken by the neonatal consultant in almost all cases (96\%), however the majority (86\%) said that there is no protocol or guideline in the unit, hence this decision is based on an individual consultant's opinion. The majority of respondents $(60 \%)$ said that they had referred $<4$ patients for sleep consultation in the last 12 months. Respondents were asked to identify clinical features that concerned them most about the possibility of SDB in a term newborn-obvious apnoea and desaturations after term gestation or multiple episodes of desaturation on overnight oximetry were the most common symptoms determining the referral (table 1).

Responses to the questions regarding management of different groups of high-risk neonates indicated that current practices are very variable. Results are described in figure 1. Practices for the use of oximetry studies also varied, from their use as a screening tool before referring for sleep consultation to how they are performed, and how the result is interpreted (table 2). The most common brand of oximeter used was Masimo. A significant proportion (36\%) of the respondents either did not know or did not reply to the question about the type of oximeter used in their unit.

\section{DISCUSSION}

In this survey of practices related to management of neonates at high risk for and/or with symptoms of SDB, we found considerable variations in screening, including the performance and interpretation of oximetry, and referral for PSG. In general, there was a shift towards PSG in infants with more severe symptoms. We suggest that there is high need for a standardised approach to the

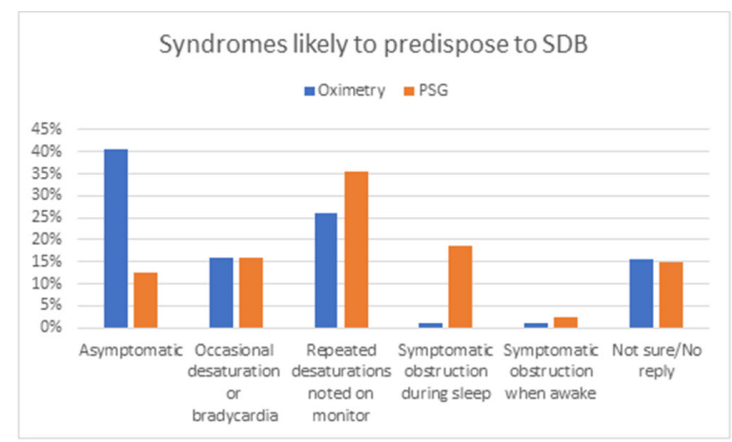

A

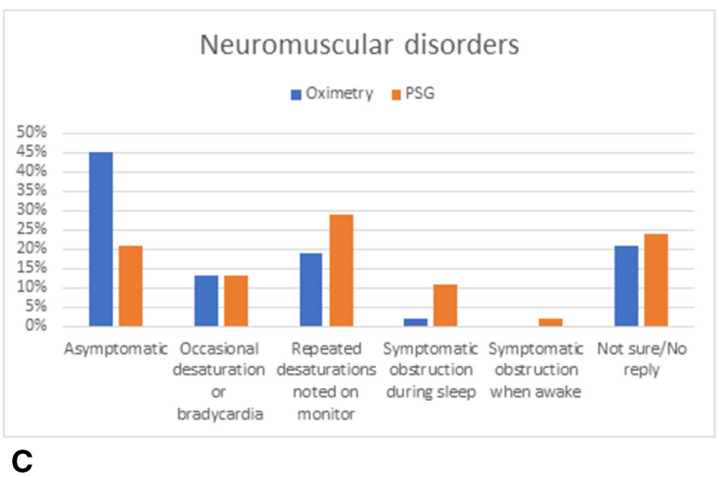

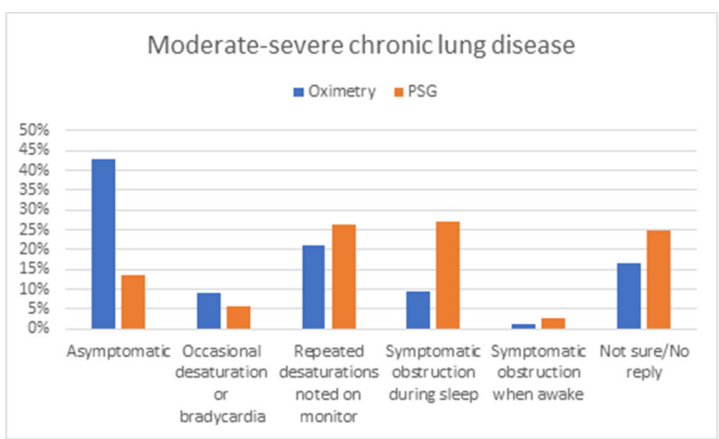

B

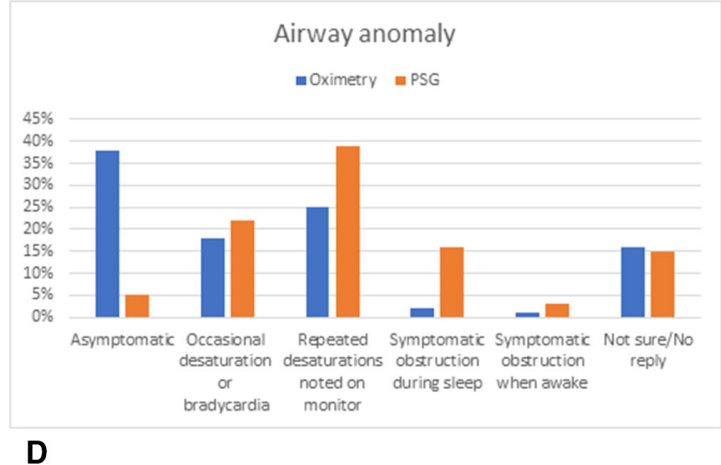

Figure 1 Management practices of neonatologists based on severity of symptoms (Likert scale-type answer option)-graphs showing at which symptom/stage they will first choose doing oximetry or refer for PSG in different conditions predisposing neonates to SDB. PSG, polysomnography; SDB, sleep disordered breathing. 
Table 2 Neonatologists' practices about use of oximetry study in neonates

\begin{tabular}{|c|c|c|}
\hline Responses & $\mathbf{N}$ & $\%$ \\
\hline \multicolumn{3}{|c|}{$\begin{array}{l}\text { 1. How often do you use an oxygen download study in your unit before referral to a sleep } \\
\text { physician? }\end{array}$} \\
\hline a. Always & 42 & 45 \\
\hline b. Usually & 13 & 14 \\
\hline c. Sometimes & 18 & 19 \\
\hline d. Never & 11 & 12 \\
\hline e. Did not answer & 9 & 10 \\
\hline \multicolumn{3}{|c|}{ 2. What is the duration of an oxygen download study in your unit? } \\
\hline a. 6 hours & 9 & 10 \\
\hline b. 8 hours & 10 & 11 \\
\hline c. 12 hours & 33 & 35 \\
\hline d. 24 hours & 32 & 34 \\
\hline e. Did not answer & 9 & 10 \\
\hline \multicolumn{3}{|c|}{ 3. What device do you use to record oximetry? } \\
\hline a. Masimo & 50 & 54 \\
\hline b. Nellcor & 9 & 10 \\
\hline c. Not sure & 16 & 17 \\
\hline d. Did not answer & 18 & 19 \\
\hline
\end{tabular}

4. What is considered abnormal oxygen download study in your unit (what features in oximetry would prompt you to refer for sleep study):

\begin{tabular}{|c|c|c|}
\hline a. Saturation $<90 \%$ for more than $5 \%$ of download & 47 & 74 \\
\hline b. Saturation $<85 \%$ for more than $2 \%$ of download & 12 & 13 \\
\hline c. Saturation $<80 \%$ for more than $1 \%$ of download & 12 & 13 \\
\hline d. Frequent bradycardia and/or desaturation & 30 & 32 \\
\hline e. Clusters of desaturations & 26 & 28 \\
\hline
\end{tabular}

management of infants with conditions associated with a high risk for and/or symptoms of SDB.

Apnoea and desaturations in a term baby were reported as the most common reasons for referral to a sleep physician. These events could be related to central apnoea due to immaturity of the central nervous system and occur commonly in healthy infants in all sleep stages. ${ }^{21}$ The frequency of central apnoea decreases with age, and prolonged or significant central apnoea is rare after 1 month of age. ${ }^{22}$ We included this question in our study as SDB in neonates is a spectrum of disorders which includes central apnoea. ${ }^{1}$ Our aim was to identify the referral pattern/threshold for referral to a sleep physician who can then decide about the need and timing for further investigation and management of apnoea whether central, obstructive or mixed.

Most respondents would perform oximetry before referring for sleep consultation. A large proportion $(35 \%-40 \%)$ of neonatologists suggested that they would order an oximetry screening study even in an asymptomatic high-risk neonate. This indicates that extended oximetry recordings are more commonly used in clinical practice to assess infants' respiratory status. However, the duration of oximetry study varied from 6 to 24 hours and there was no consensus about what is considered abnormal on an oximetry study which is consistent with previous studies. $^{2324}$

\section{Factors contributing to variations in clinical practice}

An oximetry study can be used as a surrogate measure of airway obstruction. ${ }^{25}$ However, there are limited studies so far comparing oximetry profiles with PSG parameters in neonates. ${ }^{18}$ In addition to this, one paediatric study showed that there was variation in clinical interpretation leading to different management plans when the same study report was shown to different paediatricians. ${ }^{26} \mathrm{We}$ conclude that in addition to the differences in oximeters and analysis software available for use in neonatal units, a major problem with oximetry studies is the lack of consensus guidelines for interpreting neonatal/infant oximetry reports. We think oximetry can play an important role in screening for moderate to severe OSA in high-risk infants and guide referral to a specialist centre for further evaluation. This will also help sleep physician and receiving hospital to prioritise their admission.

The levels for cut-off in various parameters in PSG to indicate pathologically significant OSA in neonates are also still being defined and little is known about the long-term 
outcome of the condition. A standardised scoring guideline with criteria for analysis of PSG in young infants $(<6$ months old) is available from the American Academy of Sleep Medicine (AASM). ${ }^{27-29}$ However, diagnosing SDB based on neonatal PSG remains challenging as normative data are not well established. Most of the previous studies of PSG involving infants have excluded neonates and/or used different criteria to define SDB such as, Respiratory Disturbance Index $>5 /$ hours of sleep ${ }^{30}$; Mixed Obstructive AHI $>2 /$ hours of sleep $^{31}$; and OSA defined as AHI $>2$ unless $>25 \%$ of events were central. ${ }^{32}$ With many studies performed prior to the publication of AASM guidelines/criteria, the generalisability of their results to the neonatal age group is restricted. ${ }^{33}$ Nonetheless, together these studies ${ }^{30-32}$ report a much higher incidence of respiratory disturbances in young infants compared with older children. A recent study ${ }^{21}$ based on AASM criteria showed that healthy newborns have a wider range of both obstructive and central events and much higher AHI than older infants and children. Thus, applying current AASM paediatric thresholds would likely result in overtreatment of sleep apnoea in neonates. This creates significant clinical dilemmas for neonatologists regarding when intervention is necessary which is further compounded by the uncertainty regarding any causal linkage between SDB and abnormal neurodevelopmental outcomes. ${ }^{11} 12$
LIMITATIONS

The main limitation of this study was low response rate. Despite efforts to improve engagement, the response rate for our survey was only $40 \%$ which could indicate selection bias. However, as responses were obtained from individuals rather than a unit-based protocol survey, we believe the result to be reasonably representative of the entire region, especially because responses include representation from across Australia and NZ. We chose to target neonatologists individually as we believed that practices by different neonatologists within a single centre may be different.

Limited feedback suggested that several respondents found this topic too difficult, making them reluctant to complete the questionnaire. Non-respondent neonatologists potentially represent people who chose not to do it due to lack of knowledge or awareness about the condition. Based on the above and considering $80 \%$ of the neonatologists who responded were working in a major NICU, we suspected selection bias meaning participation from major centres and experienced neonatologists. Therefore, the results may represent the opinions of a group of neonatologists with a higher level of knowledge than would have been present in the broader community.

Multiple participants from limited number of centres may also bias the survey results. A significant proportion

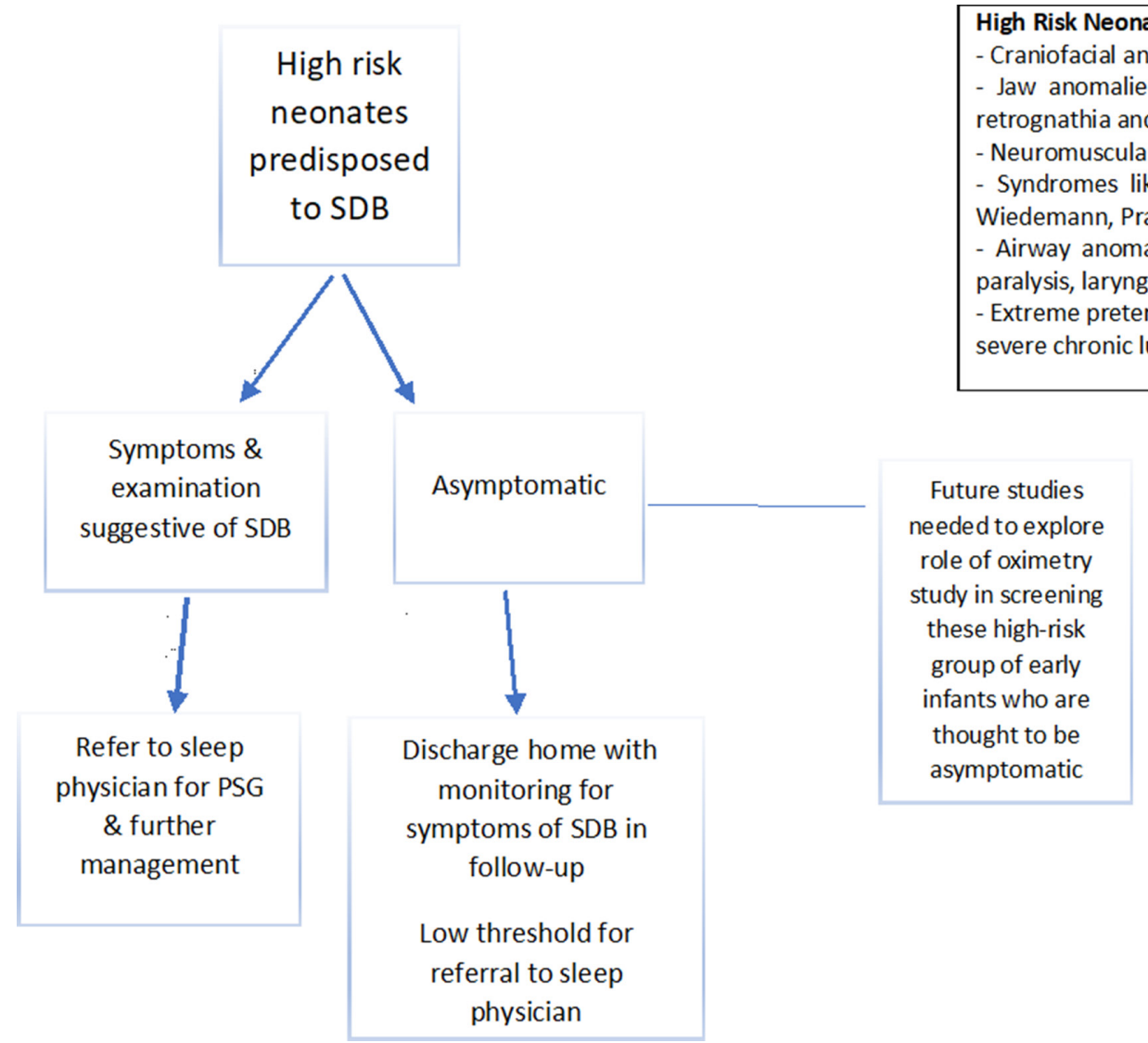

Figure 2 Suggested referral pathway for neonates suspected to have SDB. PSG, polysomnography; SDB, sleep disordered breathing. 
$(15 \%-25 \%)$ of respondents were either not sure or did not reply to the questions related to the timing of doing oximetry or referring for PSG in different clinical scenarios. Use of structured questions and answers can limit the ability of respondents to express their opinion. Providing more choices to respondents by giving them a free note option with each question can help in addressing this issue in future work. This could be achieved with a qualitative study of neonatologists' opinions on the assessment of SDB in the NICU.

In the present survey, we categorised the clinical features of SDB from being asymptomatic to having severe symptoms. The questions were designed to give the opportunity for clear answers. In reality, patients can present with combination of symptoms and signs such as oxygen desaturations with increased work of breathing which might make decision-making for the need for intervention easier for treating neonatologists.

We did not ask about the oximetry settings in this study. While this information would have been important, it was not a primary aim of the survey to specifically ask about oximetry practices. Furthermore, one-third of neonatologists could not even describe the type of oximeter used in their unit. Asking them about averaging times for data acquisition would have been even more challenging and likely raised further concerns about knowledge in this area.

\section{CONCLUSION}

This study has demonstrated a need to develop consensus guideline for screening and referring neonates with high risk for and/or symptoms of SDB. There is a need for a simple guide (figure 2) that would ensure a consistent way these high-risk neonates are managed in neonatal units throughout Australasia in an effort to decrease long-term morbidity and improve neurodevelopmental outcomes. Guidelines also need to take into account the specific condition considering the complexities and inter-related comorbidities of vulnerable infants in the NICU.

Future researchers should focus on:

- Developing a consensus guideline for the practice of neonatal oximetry studies for SDB including appropriate choice of device, settings and reporting format which are critical for interpretation.

- Identifying the prevalence of OSA in extremely low birthweight infants as they approach discharge and whether this is associated with prolonged need for supplemental oxygen.

- Identifying the consequences of SDB by correlating neonatal oximetry profiles and PSG with neurodevelopmental outcomes through early childhood and into the school-aged population.

Contributors BM participated in the development of the survey and design of the study, conducted the survey, performed data analysis and drafted the initial manuscript. KW, DF and NB participated in the conceptualisation and design of the study, reviewed and revised the manuscript, and approved the final manuscript as submitted.
Funding The authors have not declared a specific grant for this research from any funding agency in the public, commercial or not-for-profit sectors.

Competing interests None declared.

Patient consent for publication Not required.

Ethics approval Ethics approval was granted by Sydney Children's Hospital Network's Human Research and Ethics Committee-LNR/15/SCHN/470.

Provenance and peer review Not commissioned; externally peer reviewed.

Data availability statement Data are available upon reasonable request. We agree to share deidentified participant data (including data dictionaries).

Supplemental material This content has been supplied by the author(s). It has not been vetted by BMJ Publishing Group Limited (BMJ) and may not have been peer-reviewed. Any opinions or recommendations discussed are solely those of the author(s) and are not endorsed by BMJ. BMJ disclaims all liability and responsibility arising from any reliance placed on the content. Where the content includes any translated material, BMJ does not warrant the accuracy and reliability of the translations (including but not limited to local regulations, clinical guidelines, terminology, drug names and drug dosages), and is not responsible for any error and/or omissions arising from translation and adaptation or otherwise.

Open access This is an open access article distributed in accordance with the Creative Commons Attribution Non Commercial (CC BY-NC 4.0) license, which permits others to distribute, remix, adapt, build upon this work non-commercially, and license their derivative works on different terms, provided the original work is properly cited, appropriate credit is given, any changes made indicated, and the use is non-commercial. See: http://creativecommons.org/licenses/by-nc/4.0/.

\section{ORCID iD}

Bhavesh Mehta http://orcid.org/0000-0002-7099-9783

\section{REFERENCES}

1 Mehta B, Waters K, Fitzgerald D, et al. Sleep disordered breathing (SDB) in neonates and implications for its long-term impact. Paediatr Respir Rev 2020;34:3-8.

2 Bass JL, Corwin M, Gozal D, et al. The effect of chronic or intermittent hypoxia on cognition in childhood: a review of the evidence. Pediatrics 2004;114:805-16.

3 Leung LCK, Ng DK, Lau MW, et al. Twenty-Four-Hour ambulatory bp in snoring children with obstructive sleep apnea syndrome. Chest 2006;130:1009-17.

4 Landau YE, Bar-Yishay O, Greenberg-Dotan S, et al. Impaired behavioral and neurocognitive function in preschool children with obstructive sleep apnea. Pediatr Pulmonol 2012;47:180-8.

5 Gottlieb DJ, Chase C, Vezina RM, et al. Sleep-disordered breathing symptoms are associated with poorer cognitive function in 5-yearold children. J Pediatr 2004;145:458-64.

6 O'Brien LM, Mervis CB, Holbrook CR, et al. Neurobehavioral correlates of sleep-disordered breathing in children. $J$ Sleep Res 2004:13:165-72.

7 Galland BC, Dawes PJ, Tripp EG, et al. Changes in behavior and attentional capacity after adenotonsillectomy. Pediatr Res 2006;59:711-6.

8 Chervin RD, Ruzicka DL, Giordani BJ, et al. Sleep-disordered breathing, behavior, and cognition in children before and after adenotonsillectomy. Pediatrics 2006;117:e769-78.

9 Piteo AM, Kennedy JD, Roberts RM, et al. Snoring and cognitive development in infancy. Sleep Med 2011;12:981-7.

10 Smith CB, Walker K, Badawi N, et al. Impact of sleep and breathing in infancy on outcomes at three years of age for children with cleft lip and/or palate. Sleep 2014;37:919-25.

11 Bandyopadhyay A, Harmon H, Slaven JE, et al. Neurodevelopmental outcomes at two years of age for premature infants diagnosed with neonatal obstructive sleep apnea. J Clin Sleep Med 2017;13:1311-7.

12 Meerkov MS, Hassan F, Chervin RD, et al. Sleep-disordered breathing is common among term and near term infants in the NICU. Pediatr Pulmonol 2019;54:557-62.

13 Qubty WF, Mrelashvili A, Kotagal S, et al. Comorbidities in infants with obstructive sleep apnea. J Clin Sleep Med 2014;10:1213-6.

14 Ramgopal S, Kothare SV, Rana M, et al. Obstructive sleep apnea in infancy: a 7-year experience at a pediatric sleep center. Pediatr Pulmonol 2014;49:554-60.

15 Follmar A, Dentino K, Abramowicz S, et al. Prevalence of sleepdisordered breathing in patients with Beckwith-Wiedemann syndrome. J Craniofac Surg 2014;25:1814-7. 
16 Luna-Paredes C, Antón-Pacheco JL, García Hernández G, et al. Screening for symptoms of obstructive sleep apnea in children with severe craniofacial anomalies: assessment in a multidisciplinary unit. Int J Pediatr Otorhinolaryngol 2012;76:1767-70.

17 Trider C-L, Corsten G, Morrison D, et al. Understanding obstructive sleep apnea in children with charge syndrome. Int J Pediatr Otorhinolaryngol 2012;76:947-53.

18 Ehsan Z, He S, Huang G, et al. Can overnight portable pulse oximetry be used to stratify obstructive sleep apnea risk in infants? A correlation analysis. Pediatr Pulmonol 2020;55:2082-8.

19 Shellhaas RA, Kenia PV, Hassan F, et al. Sleep-Disordered breathing among newborns with myelomeningocele. J Pediatr 2018;194:244-7.

20 Huang Y-S, Hsu J-F, Paiva T, et al. Sleep-disordered breathing, craniofacial development, and neurodevelopment in premature infants: a 2-year follow-up study. Sleep Med 2019;60:20-5.

21 Daftary AS, Jalou HE, Shively L, et al. Polysomnography reference values in healthy newborns. J Clin Sleep Med 2019;15:437-43.

22 Ramanathan R, Corwin MJ, Hunt CE, et al. Cardiorespiratory events recorded on home monitors: comparison of healthy infants with those at increased risk for SIDS. JAMA 2001;285:2199-207.

23 Jamieson K, Davey MJ, Nixon GM. Oximetry knowledge in neonatal units in Victoria, Australia: can we do better? Acta Paediatr 2019;108:468-72.

24 Wellington G, Campbell AJ, Elder DE. Oximetry for preterm infants at neonatal discharge: what is current practice in New Zealand and Australia? J Paediatr Child Health 2016;52:333-7.
25 Kaditis A, Kheirandish-Gozal L, Gozal D. Pediatric OSAS: oximetry can provide answers when polysomnography is not available. Sleep Med Rev 2016;27:96-105.

26 Hardisty A, Primhak R. The interpretation of overnight oximetry tracings. Arch Dis Child 2011;96:322.

27 Berry RB, Brooks R, Gamaldo CE. For the American Academy of sleep medicine. The AASM manual for the scoring of sleep and associated events: rules, terminology and technical specifications, version 2.2. Darien, Illinois: American Academy of Sleep Medicine, 2015. www.aasmnet.org

28 Berry RB, Brooks R, Gamaldo C, et al. AASM scoring manual updates for 2017 (version 2.4). J Clin Sleep Med 2017;13:665-6.

29 Grigg-Damberger MM. The visual scoring of sleep in infants 0 to 2 months of age. J Clin Sleep Med 2016;12:429-45.

30 Ednick M, Tinkle BT, Phromchairak J, et al. Sleep-related respiratory abnormalities and arousal pattern in achondroplasia during early infancy. J Pediatr 2009;155:510-5.

31 McNamara F, Sullivan CE. Sleep-disordered breathing and its effects on sleep in infants. Sleep 1996;19:4-12.

32 Kasow KA, Stocks RMS, Kaste SC, et al. Airway evaluation and management in 7 children with malignant infantile osteopetrosis before hematopoietic stem cell transplantation. J Pediatr Hematol Oncol 2008;30:225-9.

$33 \mathrm{Ng} \mathrm{DK}$, Chan C-H. A review of normal values of infant sleep polysomnography. Pediatr Neonatol 2013;54:82-7. 